\title{
ON DAMAGE ACCUMULATION AND BIOLOGICAL AGING
}

\author{
Maxim. Finkelstein \\ Department of Mathematical Statistics \\ University of the Free State \\ PO Box 339, 9300 Bloemfontein, Republic of South Africa \\ (e-mail: FinkelM@sci.uovs.ac.za) \\ and \\ Max Planck Institute for Demographic Research, \\ Rostock, Germany
}

\begin{abstract}
If biological aging is understood as some process of damage accumulation, it does not necessarily lead to increasing mortality rate. Within the framework of suggested models and relevant examples we show that even for the monotonically increasing degradation, the mortality rate can, at least, ultimately decrease. Aging properties of systems with imperfect repair are also studied. It is shown that for some models of imperfect repair the corresponding age process is monotone and stable. This means that as $t \rightarrow \infty$, degradation slows down, which results in the mortality rate deceleration and its possible convergence to a constant.
\end{abstract}

Keywords: Aging, mortality, accumulated damage, vitality, imperfect repair

\section{INTRODUCTION}

Mortality rates of most species increase with time, at least in the post-reproductive period. Does a possible deceleration in mortality mean a deceleration in aging? This certainly depends on a definition of aging and we show under certain assumptions that when overall aging of an organism is understood as some accumulation of damage (additive degradation, which models age related dynamics of bio-markers of aging), it 
does not necessarily lead to increasing mortality rates. Therefore, we distinguish between the deterioration per se and its manifestation in the form of the increasing mortality rate, which is likely but not always to occur. In this way we partially argue with Finch (1990), where he defines senescence as "age-related changes in an organism that adversely affect its vitality and functions, but most important increase the mortality rate..." (see also Vaupel et al (2004) for the corresponding discussion). We, on the contrary, emphasize the fact that accumulation of damage of some kind, e.g., deleterious mutation accumulation (Mueller and Rose, 1996) eventually defines these age related changes in an organism and, combined with other factors, determines the shape of the mortality rate.

In sections 2-4, using our random vitality model, we analyze the shape of the mortality rate and show that even for models with monotonically increasing degradation the mortality rate can still decrease. In Section 5 aging properties of systems with imperfect repair are investigated. It turns out that for some models of imperfect repair the corresponding age process is monotone and stable. This means that as $t \rightarrow \infty$, degradation slows down, which results in the mortality rate deceleration and its possible convergence to a constant. It is worth noting that the considered stochastic approaches to mortality modeling can be used for degradation modeling in engineering systems as well.

\section{DEGRADATION AND THE SHAPE OF MORTALITY RATE}

Let $T$ denote a lifetime random variable with the cumulative distribution function (Cdf) $F(t)$ and the corresponding mortality rate $\mu(t)$. Does increasing mortality rate $\mu(t)$ really describe aging? In fact, this is a matter of definition: in reliability theory e.g., the simplest and the most popular class of aging distributions is the class of distributions with increasing failure rate (IFR) (Barlow and Proschan, 1975). The increasing mortality rate shows that probability of death of organisms in a unit interval of time increases with age. This is a rather natural definition, which certainly can be applied to human mortality at adult ages.

The foregoing expresses a statistical (black box) point of view, when the only information at hand is the mortality data. When we deal with biological aging, an underlying biological process (processes) of aging should be somehow taken into account. 
Most researchers agree that biological aging can be described by the accumulation of a damage of some kind, which leads to "age-related changes in an organism that adversely affect its vitality and functions" (Finch, 1990) and in the current note we shall follow this interpretation

Does damage accumulation (e.g., of deleterious mutations) lead to increasing mortality rates? General progressive models of aging (Aalen and Geising, 2001), described by underlying monotonically increasing stochastic processes of wear or degradation, often result in increasing mortality rates. This is, in some sense, a natural situation, but other shapes of the mortality rate are also possible.

Let, for instance, deterioration be described by the number of events $N(t), t \in[0, t)$ in the homogeneous Poisson process with rate $\lambda$. Denote by $R$ the corresponding threshold: a failure (death) occurs when the increasing $N(t)$ reaches $R$. Denote the time to failure distribution by $F(t)=\operatorname{Pr}(T \leq t)=\operatorname{Pr}(N(t) \geq R)$. It is well-known that $F(t)$ in this case is a Gamma distribution with parameters $R, \lambda$, when $R$ is an integer and $[R+1]$ otherwise. Therefore, it has an increasing mortality (failure) rate $\mu(t)$ with $\mu(0)=0$ and $\lim _{t \rightarrow \infty} \mu(t)=\lambda$.

Assume now that $R$ is random and discrete. The case of continuous $R$ will be considered in the next section. Denote by $\mu_{r}(t), r=1,2, \ldots$ the mortality rate for the realization of a threshold $r$. Then the resulting mortality rate should be obtained via the following conditioning (Yashin and Manton, 1997):

$$
\mu(t)=E\left[\mu_{R}(t) \mid T \geq t\right]
$$

Let, e.g., $R$ be Poisson-distributed with parameter $\eta$ and with additional condition that $r \geq 1$. Then

$$
P_{n}=\operatorname{Pr}(R=n)=\frac{\exp \{-\eta\} \eta^{n}}{n !(1-\exp \{-\eta\})} ; n=1,2, \ldots
$$

It can be shown (Steinsaltz and Evans, 2004) that

$$
\bar{F}(t)=\operatorname{Pr}(T>t)=\frac{1-\exp \{-\eta \exp \{-\lambda t\}\}}{1-\exp \{-\eta\}} .
$$

The corresponding mortality rate is

$$
\mu(t)=\frac{\eta \lambda \exp \{-\lambda t\}}{\exp \{\eta \exp \{-\lambda t\}\}-1},
$$


which is also an increasing function and $\lim _{t \rightarrow \infty} \mu(t)=\lambda$.

Thus the monotone degradation of a specific type leads to an increasing mortality rate in this example. The following important general result of Shaked and Shantiku$\operatorname{mar}(1988)$ is well-known:

a. An increasing jump process in a threshold model results in an increasing failure rate in average (IFRA) lifetime distribution.

b. If the process of deterioration with a threshold is described by an increasing Levy process with a Levy measure which has a decreasing density, then the corresponding lifetime is IFR.

Damage accumulation in accordance with $N(t)$ is an illustration of both of these statements. Indeed, on one hand, the homogeneous Poisson process is the specific case of a jump process. Therefore, the corresponding lifetime should be, at least, IFRA (in our specific case it is even IFR). On the other hand, the gamma process (which has a Levy measure with a decreasing density) can be viewed as a limit of the specially constructed compound Poisson process with the gamma distributed jumps, and therefore the corresponding lifetime should be IFR.

In the next section we will present some reasoning and examples which show that increasing degradation can result in decreasing (even to 0 as $t \rightarrow \infty$ ) mortality rates.

\section{RANDOM VITALITY MODEL}

Assume that at birth $(t=0)$ an organism acquires an initial unobserved random resource or vitality $R$ with the Cdf $F_{0}(r)$. Suppose that for each realization of $R$ the deterministic run out resource $W(t)(W(0)=0)$ to be called wear (or degradation) monotonically increases and continuous. Death occurs when the wear reaches $R$, which means that $W(T)=R$, therefore

$$
F(t) \equiv \operatorname{Pr}(T \leq t)=\operatorname{Pr}(W(T) \leq W(t))=\operatorname{Pr}(R \leq W(t)) .
$$

As $F_{0}(r) \equiv \operatorname{Pr}(R \leq r)$, substituting $W(t)$ instead of $r$ and taking into account equation (2):

$$
F(t)=\operatorname{Pr}(R \leq W(t))=F_{0}(W(t)) .
$$


Thus the lifetime Cdf $F(t)$ is defined in terms of the resource Cdf $F_{0}(r)$ and the wear $W(t)$. The mortality rate $\mu(t)$ can be also defined via equation (3) as

$$
\mu(t)=\frac{f(t)}{1-F(t)}=W^{\prime}(t) \lambda_{0}(W(t)),
$$

where $\lambda_{0}(t)$ is the failure rate, which corresponds to the Cdf $F_{0}(t)$ and $f(t)=F^{\prime}(t)$ is the corresponding probability density function. It is worth noting that a similar concept of a random vitality was used by Singpurwalla (2004) and was called the "hazard potential", as the role of $W(t)$ was played by the cumulated mortality rate $\int_{0}^{t} \mu(u) d u$.

Equation (4) can be used for analyzing the shape of the mortality rate in this model. Assume, at first, for simplicity that $\lambda_{0}(t)=$ const , and that $W(t)$ is increasing as a power function $t^{\alpha}, 0<\alpha<1$. It is easy to see from (4) that the mortality rate $\mu(t)$ is decreasing. Another example is the Weibull distribution with a linear failure rate: $\lambda_{0}(t)=\beta t ; \beta>0$. Then $\mu(t)$ is decreasing, when $0<\alpha<0.5$. Note, that the linear failure rate is a good approximation for the failure rate of the truncated normal distribution, which can be used for $F_{0}(r)$ modeling. Thus, these examples show that although degradation takes place (the degradation function $W(t)$ is increasing), the mortality rate is still decreasing.

It is natural to model a random wear, which is the case in reality, by a monotonically increasing stochastic process $W_{t}, t \geq 0$. Some appropriate models for $W_{t}, t \geq 0$ can be found in Singpurwala (1995). Substituting the deterministic wear $W(t)$ in (2) by the increasing stochastic process $W_{t}, t \geq 0$ leads to the following relation

$$
F(t)=\operatorname{Pr}(T \leq t)=\operatorname{Pr}\left(R \leq W_{t}\right)=E\left[F_{0}\left(W_{t}\right)\right]
$$

Similar to (1), it follows from Yashin and Manton (1997) and relation (4), that the observed mortality rate is given by the following formula

$$
\mu(t)=E\left[w_{t} \lambda_{0}\left(W_{t}\right) \mid T>t\right],
$$

where $w_{t}$ denotes the stochastic rate of diffusion: $d W_{t} \equiv w_{t} d t$ and the baseline failure rate $\lambda_{0}(t)$ is defined by the Cdf $F_{0}(t)$. 
Example 1. Let $\lambda_{0}=1$ and consider a specific case of the process of degradation: $W_{t}=w_{t}=Z k[\exp \{t\}-1]$, where $k>0$ is a constant and $Z$ is an exponential random variable with parameter $\vartheta$ (the case of a gamma $\mathrm{Cdf}$ is similar). Then, similar to Finkelstein (2003), using relation (6) it is easy to obtain by direct integration:

$$
\mu(t)=1+\frac{k-\vartheta}{k \exp \{t\}-k+\vartheta} .
$$

It follows from this equation, that $\mu(0)=k / \vartheta$. For $k<\vartheta, \mu(t)$ is monotonically increasing, asymptotically converging to 1 , whereas for $k>\vartheta$ it is monotonically decreasing.

Example 2. As in the previous example, consider a specific multiplicative degradation model : $W_{t}=Z t$, where $Z$ has a Weibull distribution with parameters $\lambda$ (scale) and $R$ (shape). Assume also that the threshold $R$ is deterministic. Then by direct reasoning:

$$
F(t)=\operatorname{Pr}(Z t \geq R)=\operatorname{Pr}\left(Z \geq \frac{R}{t}\right)=\exp \left\{-\left(\frac{\lambda R}{t}\right)^{k}\right\}
$$

and the corresponding mortality rate is

$$
\mu(t)=\frac{k(\lambda k)^{k} t^{-k-1} \exp \left\{-\left(\frac{\lambda R}{t}\right)^{k}\right\}}{1-\exp \left\{-\left(\frac{\lambda R}{t}\right)^{k}\right\}} .
$$

It is easy to see that $\lim _{t \rightarrow 0} \mu(t)=\lim _{t \rightarrow \infty} \mu(t)=0$ (Bae et al, 2007) and that this function is monotonically increasing in $\left(0, t_{\max }\right)$ and then monotonically decreasing in $\left[t_{\max }, \infty\right)$, where $t_{\max }$ denotes the value of $t$ at which it reaches the maximum. Thus the shape of $\mu(t)$ exhibits the ultimate decline to 0 , although degradation is modeled by the increasing paths stochastic process.

Finally it is worth mentioning that if the process of wear $\left\{W_{t}, t \geq 0\right\}$ is modeled by the Wiener process with drift, then the first-passage problem for the deterministic threshold $R$ results in the inverse Gaussian time to failure distribution with ultimately 
decreasing or increasing (which depends on the values of parameters involved) mortality rate.

\section{GENERALIZATION OF THE STREHLER-MILDVAN MODEL}

As in the previous model, consider a first passage-type setting but with an additional feature of killing events (Singpurwalla, 1995; Finkelstein 2003): Let $W_{t}, t \geq 0$ denote an increasing stochastic process of damage accumulation and let $B(t)$ be a deterministic function that defines the corresponding boundary. Death of an organism occurs when $W_{t}$ exceeds $B(t)$ for the first time.

Let $P_{t}, t \geq 0$ be a point process of shocks with rate $\lambda(t)$, which is independent from $W_{t}, t \geq 0$. In the frame of the Strehler-Mildvan (1960) model we understand shocks as external instantaneous demands for energy. Assume for simplicity that probability of $W_{t}$ reaching the boundary is negligible. Therefore shocks can be considered as the only cause of death. Assume also that independently from the history, each shock causes death with probability $\theta(t)$ and is 'survived' with the complementary probability $1-\theta(t)$. This can be interpreted in the following way: each shock has a random magnitude $Y$ with a common distribution function $\Psi(y)$. The death at age $t$ occurs when this magnitude exceeds $B(t)-W(t)$, where $W(t)$ is a realization of $W_{t}, t \geq 0$. Therefore, e.g., for realizations

$$
\theta(t)=\operatorname{Pr}(Y>B(t)-W(t) \mid T \geq t)=1-\Psi(B(t)-W(t)) .
$$

In the original Strehler-Mildvan (1960) model, which was widely applied to human mortality data, our $B(t)-W(t)$ had a meaning of remaining vitality at time $t$. It was also supposed in this model that this function linearly decreases with age, which can be a reasonable assumption as some biological markers of human aging can behave linearly (Nakamura et al, 1998). But the crucial unjustified assumption was that the distribution function $\Psi(y)$ is exponential (Yashin et al, 2000). The combination of linearity of $B(t)-W(t)$ and of exponentiality of $\Psi(y)$ had resulted in the exponential form of the mortality rate and therefore can not be considered as a justification of the empirical Gompertz law of human mortality. 
Denote by $\lambda\left(t, H_{t}\right)$ the complete intensity function of the orderly point process $P_{t}, t \geq 0$ (Cox and Isham, 1980), which takes into account the history of the process up to time $t$. Thus, $\lambda\left(t, H_{t}\right) d t$ can be interpreted as a probability of a shock occurrence in $[t, t+d t)$, given the process history up to $t$. In accordance with our simplifying assumption: $\theta\left(t, H_{t}\right) \equiv \theta(t)$ and therefore the conditional mortality rate in our model is:

$$
\begin{aligned}
& \mu_{c}\left(t, H_{t}\right) d t=\operatorname{Pr}\left\{T \in[t, t+d t) \mid H_{t}, T\left(H_{t}\right) \geq t\right\} \\
& =\theta(t) \lambda\left(t, H_{t}\right) d t
\end{aligned}
$$

where condition $T\left(H_{t}\right) \geq t$ means that all shocks in $[0, t)$ were survived. It is clear, that only for the specific case of the Poisson process:

$$
\mu_{c}\left(t, H_{t}\right)=\theta(t) \lambda(t)=\mu(t)
$$

and the corresponding survival function is

$$
\bar{F}(t) \equiv 1-F(t)=\exp \left\{-\int_{0}^{t} \theta(u) \lambda(u) d u\right\}
$$

Unfortunately, Strehler-Mildvan (1960) did not make this crucial assumption, without which their approach is not mathematically valid.

Equation (9) states that the resulting mortality rate is just a simple product of the rate of the Poisson process and of the probability $\theta(t)$. Therefore, its shape can be easily analyzed. When, e.g., $B(t)-W(t)$ is decreasing, the probability $\theta(t)$ is increasing with age, which goes in line with the accumulation of degradation reasoning. If, additionally, the rate of harmful events $\lambda(t)$ is not decreasing, the resulting mortality rate $\mu(t)$ is also increasing. On the other hand, other relevant scenarios resulting in decreasing mortality rate are also possible (Vaupel and Yashin, 1987, Finkelstein, 2005).

\section{AGING OF REPAIRABLE SYSTEMS}

In previous sections we considered an overall process of degradation in organisms and the corresponding mortality (death) rates. But this deterioration results from degradation processes on the lower, e.g., cell level. According to a number of authors (see Yashin et al. (2000) for references) the DNA repair capacity can be responsible for 
aging of humans. One can assume that in the absence of the proper (perfect) repair the spontaneous DNA mutations result in aging and eventually in death of an organism. Thus imperfect repair is likely to be the cause of various aging processes. In what follows we define aging in repairable systems and show that certain types of imperfect repair can still lead to the defined asymptotic "non-aging" property.

Consider some hypothetical repairable object, to be called for convenience a component, which starts functioning at $t=0$. Assume, as usually in renewal theory, that repair is perfect: after repair a component is as good as new. Then the i.i.d. sequence of inter-arrival times $\left\{T_{i}\right\}_{i \geq 1}$ with a common distribution function $F(t)$ forms a standard renewal process. The renewal (waiting) times in this case are given by the sequence $S_{0}=0, S_{1}=T_{1}, S_{2}=T_{1}+T_{2}, S_{3}=T_{1}+T_{2}+T_{3}, \ldots$ The corresponding stochastic intensity in this case is compactly written via the indicator function as

$$
\lambda_{t}=\sum_{n=0}^{\infty} \lambda\left(t-S_{n}\right) I\left(S_{n} \leq t<S_{n+1}\right) ; t \geq 0,
$$

where, as usual, $\lambda(t)=F^{\prime}(t) / \bar{F}(t)$. Denote by $A_{t}$ the age process, which corresponds to the renewal process (11):

$$
A_{t}=\sum_{n=0}^{\infty}\left(t-S_{n}\right) I\left(S_{n} \leq t<S_{n+1}\right) ; t \geq 0 .
$$

Assume that the generic $F(t) \in I F R$, which means that the corresponding failure rate $\lambda(t)$ is not decreasing. Therefore $F(t)$ is an aging distribution. It is clear also that the perfect repair does not lead to accumulation of damage. But this is not so when the repair is not perfect, which is the case in nature and in most technical systems

There are two major possibilities. The first one is when the imperfect repair reduces wear only of the last cycle. It is clear that in this case the overall wear increases and under some reasonable assumptions this operation only decreases the rate of accumulation of wear for the process. This ant-aging mechanism is described in Finkelstein (2003). Situation starts to be much more interesting when the current repair reduces the overall accumulated wear. We shall model this setting in the following way: Assume that the first instantaneous repair at $t=t_{1}$ (realization of $T_{1}$ ) decreases the age of a system not to 0 as in the case of a perfect repair, but to $v_{1}=q t_{1}, 0<q<1$, and the system starts the second cycle with this initial age in accordance with the dis- 
tribution of the remaining lifetime $1-\bar{F}\left(v_{1}+t\right) / \bar{F}\left(v_{1}\right)$. The constant $q$ defines the quality of repair.

Thus, the reduction of wear is modelled by the corresponding reduction in age after the repair. Note that, as the failure rate of a component $\lambda(t)$ is increasing, the described operation also decreases its value and the failure rate at the beginning of the new cycle in this realization is smaller, than it was at the end of the previous one. The forthcoming cycles are defined in a similar way to form a process of general repair (Kijima, 1989; Finkelstein, 2000). The sequence of ages after the $i$ th repair $\left\{V_{i}\right\}_{i \geq 0}$ in this model is defined as

$$
V_{0}=0 ; V_{1}=q T_{1} ; V_{2}=q\left(V_{1}+T_{2}\right), \ldots, V_{i}=q\left(V_{i-1}+T_{i}\right), \ldots
$$

and distributions of the corresponding inter-arrival times for realizations $v_{i}$ are given by:

$$
\bar{F}_{i}(t)=\frac{\bar{F}\left(v_{i-1}+t\right)}{\bar{F}\left(v_{i-1}\right)}, i \geq 1 .
$$

Denote the distribution of age at the start of the $(i+1)$ th cycle by $A_{i+1}^{S}(v)$, $i=1,2, \ldots . \quad\left(v=0\right.$ at the start of the first cycle) and by $A_{i}^{E}(v), i=1,2, \ldots$ the corresponding age distribution at the end of the previous $i$ th cycle. It is clear that in accordance with our model (12):

$$
A_{i+1}^{S}(v)=A_{i}^{E}(v / q), i=1,2, \ldots
$$

The proof of following results can be found in Finkelstein (2007):

a. Random ages at the end (start) of each cycle in the general repair model (12) form a stochastically increasing sequences:

$$
\bar{A}_{i+1}^{E}(v)>\bar{A}_{i}^{E}(v),\left(\bar{A}_{i+2}^{S}(v)>\bar{A}_{i+1}^{S}(v)\right), t>0 ; v>0, i=1,2, \ldots .
$$

b. There exist limiting distributions for ages at the start and at the end of cycles:

$$
\lim _{i \rightarrow \infty} A_{i}^{E}(v)=A_{L}^{E}(v),\left(\lim _{i \rightarrow \infty} A_{i}^{S}(v)=A_{L}^{S}(v)\right) .
$$

The interpretation of this result is very simple and meaningful. The described renewal-type process is aging, because the ages at the start (end) of the cycles are sto- 
chastically increasing with $i$ and the generic failure rate $\lambda(t)$ is increasing. On the other hand, it follows from (14) that the sequences of ages have a finite limit, which means that aging slows down and asymptotically vanishes with $i \rightarrow \infty$.

If the repair process in parts of organisms decreases the accumulated wear and not only the wear of the last cycle, then the mortality rate (as a function of degradation) of these parts and of an organism as a whole, slows down at advanced ages and can even tend to a constant (mortality plateau). Therefore, our model can explain the deceleration of human mortality at advanced ages (see, e.g., Thatcher (1999)) and even approaching a mortality plateau.

\section{Acknowledgements}

The author wants to thank two anonymous referees for extremely helpful comments.

\section{References}

Aalen, O.O., and Gjessing, H.K. (2001). Understanding the shape of the hazard rate: a process point of view. Statistical Science, 16: 1-22.

Bae, S, Kuo, W., and Kvam, P. Degradation models and implied lifetime distributions. Reliability Engineering and System Safety, to appear.

Barlow R., and Proschan F. (1975). Statistical Theory of Reliability and Life Testing. Probability Models. New-York: Holt, Rinehart and Winston.

Cox D.R., Isham V. (1980). Point Processes, Chapman and Hall.

Finch, C (1990). Longevity, Senescence, and the Genome. University of Chicago Press, Chicago.

Finkelstein, M.S. (2000). Modeling a process of non-ideal repair. In: Recent Advances in Reliability Theory. Limnios N., Nikulin M. (eds). Birkhauser, 41-53.

Finkelstein, M.S. (2003). A model of biological aging and the shape of the observed hazard rate. Lifetime Data Analysis, 9: 93-109.

Finkelstein, M.S. (2005). Lifesaving explains mortality decline with time. Mathematical Biosciences, 196/2, 187-197.

Finkelstein, M.S. (2007). On some aging properties of general repair processes. Journal of Applied Probability, 44, 506-513.

Kijima, M. (1989). Some results for repairable systems with general repair. Journal of Applied Probability, 26, 89-102. 
Mueller, L..D., and Rose, M.R. (1996). Evolutionary theory predicts late life mortality plateaus. PNAS, 93, 15249-15243.

Nakamura, E., Lane, M., Roth, G., Ingram, D. (1998). “A strategy for identifying biomarkers of aging”. Exp. Gerontology, 33: 4.

Singpurwalla, N. D. (1995). Survival in dynamic environment. Statistical Science, 10, 86-108.

Singpurwalla, N. D. (2004). The hazard potential of items and individuals. Technical Report. The George Washington University.

Shaked, M., and Shantikumar, J.G. (1988). On the first-passage time of the pure jump process. Journal of Applied Probability, 25, 501-509.

Strehler, B.L., and Mildvan, A. S. (1960). General theory of mortality and aging. Science, 132, 14-21.

Steinsaltz, D., and Evans, S. (2004). Markov mortality models: Implications of quasistationarity and varying initial distributions. Theoretical Population Biology, 65:4, June.

Thatcher, A.R. (1999) "The long-term pattern of adult mortality and the highest attained age." J. R. Statist. Soc. A, vol. 162, pp. 5-43.

Vaupel, J.W., and Yashin, A.I. (1987). Repeated resuscitation: how life saving alters

life tables. Demography, 4, 123-135

Yashin, A. I., and K.G. Manton, K. G. (1997). Effects of unobserved and partially observed covariate processes on system failure: a review of models and estimation strategies. Statistical Science, 12, 20-34.

Yashin, A. I, Iachine, I.A., and Begun, A. S. (2000). Mortality modeling: a review. Mathematical Population Studies, 8: 305-332. 